\title{
New Avian Hepadnavirus in Palaeognathous Bird, Germany
}

\section{Wendy K. Jo, ${ }^{1}$ Vanessa M. Pfankuche, ${ }^{1}$ Henning Petersen, Samuel Frei, Maya Kummrow, Stephan Lorenzen, Martin Ludlow, Julia Metzger, Wolfgang Baumgärtner, Albert Osterhaus, Erhard van der Vries}

Author affiliations: University of Veterinary Medicine Hannover, Foundation, Hannover, Germany (W.K. Jo, V.M. Pfankuche, H. Petersen, M. Ludlow, J. Metzger, W. Baumgärtner, A. Osterhaus, E. van der Vries); Center for Systems Neuroscience, Hannover (W.K. Jo, V.M. Pfankuche, W. Baumgärtner, A. Osterhaus); Wuppertal Zoo, Wuppertal, Germany (S. Frei, M. Kummrow); Bernhard Nocht Institute for Tropical Medicine, Hamburg (S. Lorenzen); Artemis One Health, Utrecht, the Netherlands (A. Osterhaus)

DOI: https://doi.org/10.3201/eid2312.161634

In 2015, we identified an avian hepatitis B virus associated with hepatitis in a group of captive elegant-crested tinamous (Eudromia elegans) in Germany. The full-length genome of this virus shares $<76 \%$ sequence identity with other avihepadnaviruses. The virus may therefore be considered a new extant avian hepadnavirus.

$\mathrm{H}^{2}$ epatitis B virus (HBV) belongs to the family Hepadnaviridae, members of which constitute 2 major extant genera: Orthohepadnavirus, which infect mammals, and Avihepadnavirus, which infect birds (1). Recently, evidence of a likely third genus was found with the discovery of a new fish hepadnavirus (2). In addition, HBV-derived endogenous viral elements have been reported in several neoavian birds (e.g., budgerigars and several finches) $(3,4)$ and reptiles (e.g., turtles and crocodiles) (5).

Hepadnaviruses generally are characterized by their narrow host range and strong hepatotropism (1). They are enveloped, partially double-stranded DNA viruses with a small circular genome $(\approx 3 \mathrm{~kb})$ and at least 3 open reading frames (ORFs) (1). In orthohepadnaviruses, a fourth ORF encodes the $\mathrm{X}$ protein, which is associated with hepatocellular carcinoma in their respective host species. Avihepadnaviruses appear to have an X-like protein region; however, either a premature stop codon is present or no ORF is found in most cases (6). We describe a new avian HBV causing severe hepatitis in the elegant-crested tinamou (Eudromia elegans), a member of the ancient group of birds the Palaeognathae, which

${ }^{1}$ These authors contributed equally to this article. includes emus (Dromaius novaehollandiae) and ostriches (Struthio spp.).

In 2015, a deceased adult elegant-crested tinamou kept at Wuppertal Zoo (Wuppertal, Germany) underwent necropsy at the University of Veterinary Medicine Hannover, Foundation (Hannover, Germany). Initial histologic examination revealed moderate, necrotizing hepatitis and inclusion body-like structures within the hepatocytes. To identify a putative causative agent, we isolated nucleic acids from the liver and prepared them for sequencing on an Illumina MiSeq system (Illumina, San Diego, CA, USA) (online Technical Appendix, https://wwwnc.cdc.gov/EID/ article/23/12/16-1634-Techapp1.pdf). We compared obtained reads with sequences in GenBank using an in-house metagenomics pipeline. Approximately $78 \%$ of the reads aligned to existing avihepadnavirus sequences. A full genome $(3,024 \mathrm{bp})$ of the putative elegant-crested tinamou HBV (ETHBV) was subsequently constructed by de novo assembly mapping $>2$ million reads $(88.6 \%)$ to the virus genome (GenBank accession no. KY977506).

The newly identified ETHBV shared $<76 \%$ nt sequence identity with other avian HBVs (online Technical Appendix Table 1). Phylogenetic analysis showed that ETHBV clustered within the genus Avihepadnavirus, forming a new clade (Figure, panel A). The organization of the ETHBV genome was similar to other avian HBVs because all 3 overlapping ORFs (polymerase, nucleocapsid [preC/C], and presurface $[\mathrm{preS} / \mathrm{S}]$ antigen) and several essential sequence motifs (e.g., the epsilon element, TATA boxes, and direct repeat sites DR1 and DR2) were identified (online Technical Appendix Figure 1). We also found an X-like sequence. However, similar to duck HBV, ETHBV lacks a putative translation start site. It has been suggested that the $\mathrm{X}$ protein evolved later in mammalian hosts (5), which explains the absence of X-like ORF in the ETHBV genome. Comparison of pairwise amino acid identities between ETHBV and other avihepadnaviruses showed low homologies between their functional proteins $(64 \%-69 \%$ similarity to the polymerase, $75 \%-80 \%$ to the $\mathrm{preC} / \mathrm{C}, 52 \%-62 \%$ to the preS/S [online Technical Appendix Table 2]).

The identification of ETHBV prompted us to retrospectively screen the flock of 7 elegant-crested tinamous at Wuppertal Zoo and the 6 that had died within the past 4 years and had undergone necropsy at the University of Veterinary Medicine Hannover, Foundation (online Technical Appendix Table 4). For that purpose, we designed a set of degenerated primers targeting a short region of the polymerase-preC/C genome in all avihepadnaviruses (online Technical Appendix). All birds were found positive by PCR (online Technical Appendix Table 4), including liver tissue from embryonated eggs, implying that ETH$\mathrm{BV}$ is vertically transmitted (Figure, panel B). We then obtained a second ETHBV genome (GenBank accession 
A
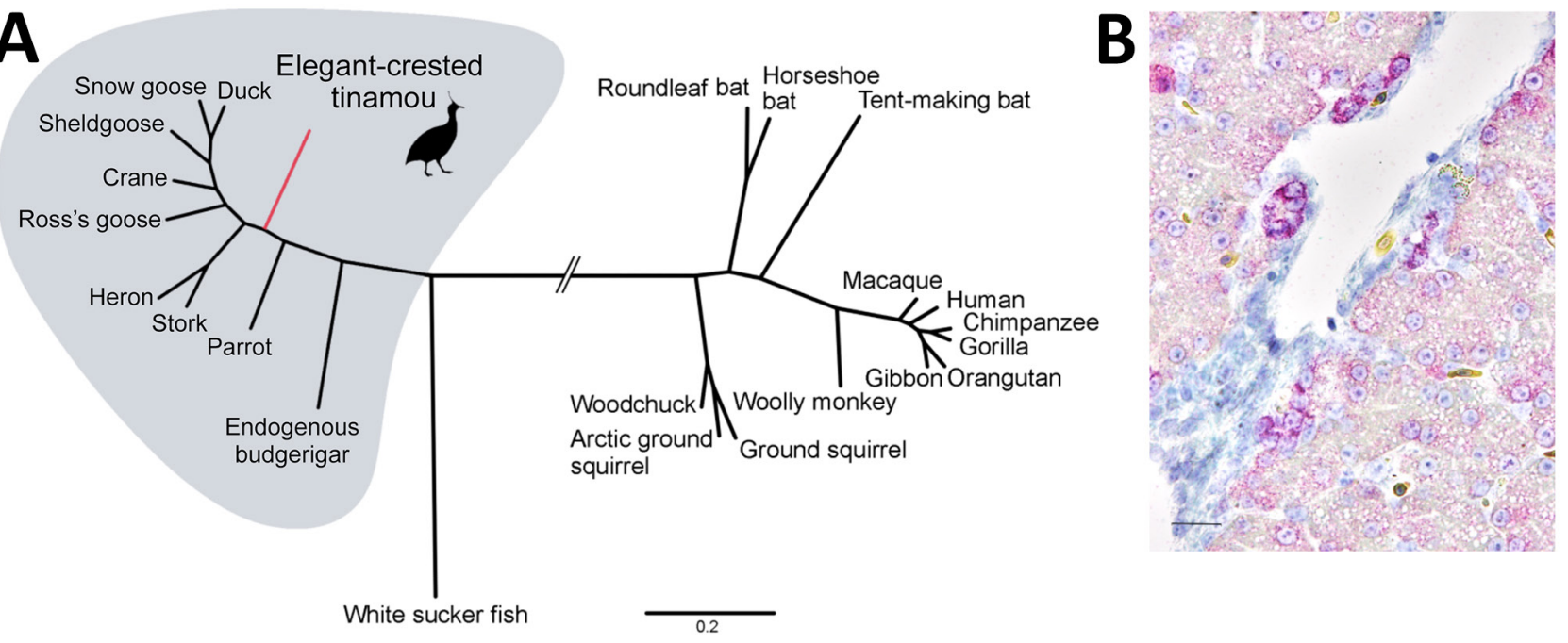

Figure. Phylogenetic and histopathologic analysis of probable new avian hepadnavirus, elegant-crested tinamou hepatitis $B$ virus (ETHBV), Germany. A) Bayesian phylogeny of virus isolated from an elegant-crested tinamou (Eudromia elegans) compared with reference viruses. Tree was created on the basis of full-genome sequences from the family Hepadnaviridae. The analysis was run for 4 million generations and sampled every 100 steps, and the first $25 \%$ of samples were discarded as burn-in in MrBayes (7). HasegawaKishino-Yano nucleotide substitution model was selected as best-fit model according to Bayesian information criteria. Posterior probabilities are shown in online Technical Appendix Figure 3 (https://wwwnc.cdc.gov/EID/article/23/12/16-1634-Techapp1.pdf). Branches were truncated for graphical reasons (interrupted lines). GenBank accession number for isolates, is available online (http://wwwnc.cdc.gov/EID/article/23/16-1634-F1.htm). Scale bar indicates nucleotide substitutions per site. B) ETHBV-specific RNA (in red; Fast Red) localized within hepatocytes of the liver tissue of an elegant-crested tinamou embryo by in situ hybridization (online Technical Appendix). Positive signal is enhanced in hepatocytes localized close to the vessels and negative in endothelial cells.

Nonprobe incubation of the tinamou and liver tissue from a pheasant were used as negative controls. Scale bar indicates $40 \mu \mathrm{m}$.

no. KY977507) from another tinamou from the same flock by deep sequencing; this genome showed $99.8 \% \mathrm{nt}$ sequence identity with the initial ETHBV genome. Tinamou serum samples from another zoo were also screened but tested negative by PCR (online Technical Appendix Table 4).

To further characterize ETHBV, we confirmed infection in the liver using an in situ hybridization protocol $(8)$ in an adult and embryo tinamou (Figure, panel B). In addition to ETHBV infection in the liver, we found some positive cells in kidney and testis tissue. Although hepadnaviruses generally are host restricted, exceptions have been reported (e.g., crane HBV) (9). We attempted to infect Pekin duck embryos through the allantoic cavity, as well as by intravenous infection routes, and were not able to demonstrate replication (data not shown).

ETHBV can be considered a new extant hepadnavirus associated with hepatitis in the elegant-crested tinamou. Whether ETHBV can infect other species within the Palaeognathae or whether it is host restricted within other tinamou species remains to be elucidated. The discovery of ETHBV suggests that other avian species may harbor as-yet undiscovered HBVs. The pathogenesis of avian hepadnavirus infections and the mechanisms of virus transmission in captive tinamou flocks warrant further investigation.

\section{Acknowledgments}

We thank Mareike Schubert, Kerstin Rohn, Danuta Waschke, Bettina Buck, Caroline Schütz, Kerstin Schöne, Heike KlippertHasberg, Mogens Drabert, Stefan Neander, and Jörn Wrede for excellent technical assistance. We also are grateful to Severin Dressen and Emile Prins for their collaboration in the project.

This study was in part supported by a grant from the Niedersachsen-Research Network on Neuroinfectiology from the Ministry of Science and Culture of Lower Saxony, Germany. This study also was in part supported by the COMPARE project and received funding from the European Union's Horizon 2020 research and innovation program COMPARE (grant agreement no. 643476).

Dr. Jo is a PhD candidate at the University of Veterinary Medicine Hannover Research Center for Emerging Infections and Zoonoses, Hannover, Germany. Her research interests include virus discovery, emerging and re-emerging infections, cross-species transmission, viral evolution, and host adaptation.

\section{References}

1. Funk A, Mhamdi M, Will H, Sirma H. Avian hepatitis B viruses: molecular and cellular biology, phylogenesis, and host tropism. World J Gastroenterol. 2007;13:91-103. http://dx.doi.org/10.3748/ wjg.v13.i1.91 
2. Hahn CM, Iwanowicz LR, Cornman RS, Conway CM, Winton JR, Blazer VS. Characterization of a novel hepadnavirus in the white sucker (Catostomus commersonii) from the Great Lakes region of the United States. J Virol. 2015;89:11801-11. http://dx.doi.org/ 10.1128/JVI.01278-15

3. Liu W, Pan S, Yang H, Bai W, Shen Z, Liu J, et al. The first fulllength endogenous hepadnaviruses: identification and analysis. J Virol. 2012;86:9510-3. http://dx.doi.org/10.1128/JVI.01164-12

4. Suh A, Brosius J, Schmitz J, Kriegs JO. The genome of a Mesozoic paleovirus reveals the evolution of hepatitis B viruses. Nat Commun. 2013;4:1791. http://dx.doi.org/10.1038/ncomms2798

5. Suh A, Weber CC, Kehlmaier C, Braun EL, Green RE, Fritz U, et al. Early mesozoic coexistence of amniotes and hepadnaviridae. PLoS Genet. 2014;10:e1004559. http://dx.doi.org/10.1371/ journal.pgen.1004559

6. Chang SF, Netter HJ, Hildt E, Schuster R, Schaefer S, Hsu YC, et al. Duck hepatitis B virus expresses a regulatory $\mathrm{HBx}$-like protein from a hidden open reading frame. J Virol. 2001;75: 161-70. http://dx.doi.org/10.1128/JVI.75.1.161-170.2001

7. Huelsenbeck JP, Ronquist F. MRBAYES: Bayesian inference of phylogenetic trees. Bioinformatics. 2001;17:754-5. http://dx.doi.org/10.1093/bioinformatics/17.8.754

8. Pfankuche VM, Bodewes R, Hahn K, Puff C, Beineke A, Habierski A, et al. Porcine bocavirus infection associated with encephalomyelitis in a pig, Germany. Emerg Infect Dis. 2016;22:1310-2. http://dx.doi.org/10.3201/eid2207.152049

9. Prassolov A, Hohenberg H, Kalinina T, Schneider C, Cova L, Krone O, et al. New hepatitis B virus of cranes that has an unexpected broad host range. J Virol. 2003;77:1964-76. http://dx.doi.org/10.1128/JVI.77.3.1964-1976.2003

Address for correspondence: Erhard van der Vries, Department of Infectious Diseases \& Immunology, Faculty of Veterinary Medicine, Section Virology, Utrecht University, Utrecht, the Netherlands; email: e.vandervries@uu.nl

\section{Acute Myopericarditis Associated with Tickborne Rickettsia sibirica mongolitimonae}

\section{Pablo Revilla-Martí, Álvaro Cecilio-Irazola, Jara Gayán-Ordás, Isabel Sanjoaquín-Conde, Jose Antonio Linares-Vicente, José A. Oteo}

Author affiliations: Hospital Clínico Universitario Lozano Blesa,

Zaragoza, Spain (P. Revilla-Martí, A. Cecilio-Irazola,

J. Gayán-Ordás, I. Sanjoaquín-Conde, J.A. Linares-Vicente);

Centro de Investigación Biomédica de La Rioja, Logroño, Spain

(J.A. Oteo)

DOI: https://doi.org/10.3201/eid2312.170293
We report an unusual case of myopericarditis caused by Rickettsia sibirica mongolitimonae. Because of increasing reports of Rickettsia spp. as etiologic agents of acute myopericarditis and the ease and success with which it was treated in the patient reported here, rickettsial infection should be included in the differential diagnosis for myopericarditis.

$\mathrm{M}^{\mathrm{s}}$ yopericarditis is a primarily pericardial inflammatory syndrome occurring when clinical diagnostic criteria for pericarditis are satisfied and concurrent mild myocardial involvement is documented by elevated biomarkers of myocardial damage (i.e., increased troponins). Limited clinical data on the causes of myopericarditis suggest that viral infections are among the most common causes in developed countries, although the list of agents is increasing. We identified an unusual case of myopericarditis caused by Rickettsia sibirica mongolitimonae, an emerging pathogen in southern Europe with a broad clinical spectrum (1).

In September 2016, a 39-year-old man with no remarkable medical history sought care at an emergency department in Spain with acute-onset central chest pain and fever. The previous week, he had hunted in northeastern Spain. Physical examination revealed a systolic blood pressure of $115 \mathrm{~mm} \mathrm{Hg}$, heart rate 80 beats/min, peripheral pulse oximetry of $98 \%$, and an axillary temperature of $38.7^{\circ} \mathrm{C}$. No murmurs, rales, or gallops were detected on cardiac examination. A necrotic left gluteus eschar and multiple enlarged left inguinal lymph nodes were noted. He had neither lymphangitis nor widespread rash, and his mucous membranes appeared normal. He did not remember tick bites.

An electrocardiogram demonstrated a sinus rhythm with diffuse ST-segment elevation, and a transthoracic echocardiogram showed a normal biventricular ejection fraction with mild pericardial effusion. High-sensitive $T$ troponin level was $575.3 \mathrm{ng} / \mathrm{L}$ (reference $<14 \mathrm{ng} / \mathrm{L}$ ), and blood cultures and serologic tests for common viruses were all negative. He was admitted to the hospital, and a cardiac magnetic resonance study performed 48 hours later confirmed the suspected diagnosis of myopericarditis.

Because of the eschar, tickborne-related rickettsiosis was suspected, and ibuprofen $(1,800 \mathrm{mg} / \mathrm{d})$ and doxycycline (100 mg every $12 \mathrm{~h}$ ) were started. After the third day on medical therapy, the patient became afebrile, and the electrocardiographic changes gradually resolved. He was discharged after 12 days. Doxycycline was maintained for 14 days.

Acute-phase serologic tests yielded negative results for HIV; Borrelia burgdorferi sensu lato (chemiluminiscence immunoassay, Liason, Diasorin, Spain); spotted fever group rickettsia (SFGR) (commercial [Focus Diagnostics, Cypress, CA, USA] and in-house tests); and Francisella tularensis (in-house microagglutination assay). An eschar swab sample and an eschar biopsy sample were removed under aseptic 